\title{
Are renewables affecting income distribution and increasing the risk of household poverty?
}

\author{
Diogo Santos Pereira ${ }^{\mathrm{a}}$, António Cardoso Marques ${ }^{\mathrm{b}, *}$, José Alberto Fuinhas \\ ${ }^{a}$ University of Beira Interior, Management and Economics Department; \\ ${ }^{b}$ NECE-UBI ${ }^{1}$, University of Beira Interior, Rua Marquês d'Ávila e Bolama, \\ 6201-001 Covilhã, Portugal Tel. + 351275319600 Fax. + 351275319601 \\ *Corresponding author: amarques@ubi.pt; acardosomarques@gmail.com
}

\begin{abstract}
The worldwide electricity mix has become diversified, mainly through the exploitation of endogenous and green resources. However, doubt has been cast on the much-vaunted advantages of renewables due to some of their characteristics, such as availability, security and affordability. In fact, growth in the installed capacity of renewable energy has increased electricity prices, which raises the question of how households have withstood the cost of energy transition. The main aim of this study is to empirically assess and discuss: (i) whether different types of household have suffered dissimilar effects from the promotion of renewables; (ii) the consequences of promoting renewables on household income; and (iii) if the promotion of renewables has reduced the risk of poverty and social exclusion. A panel data of European countries has been analysed using Kao's residual cointegration test, and an Autoregressive Distributed Lag approach, to assess the relationships. This paper proves that both income and risk of household poverty are directly linked with renewable energies, in both the short- and long-run. The energy transition to renewables has had negative consequences for households. Thus, the disadvantaged households should be helped to meet the increased cost arising from the energy transition.
\end{abstract}

\section{Keywords:}

Renewable energy sources; energy transition; electricity prices; income redistribution effects; risk of poverty

\section{Highlights:} households.

The impact of deploying renewables on income and the risk of poverty for

The Kao test verifies the long-run relation between renewables and risk of poverty.

The ARDL technique takes into consideration both short- and long-run effects. Solar PV deployment has increased the overall risk of poverty for households.

\footnotetext{
${ }^{1}$ The financial support of the NECE-UBI, Research Unit in Business Science and Economics, sponsored by the Portuguese Foundation for the Development of Science and Technology, project UID/GES/04630/2013, is gratefully acknowledged.
} 
Policies are required to mitigate the negative effects of RES on society.

\section{Introduction}

The world's population has been growing and developing, increasing the demand for energy, particularly electrical energy. Electricity is considered a key energy source for the future, playing a fundamental role in socio-economic and sustainable development. In the near future, it is expected that the residential, industrial, services, transport, and heating sectors will only consume electricity, as will public services such as education, health, and sanitation. It is hoped that cleaner, green electricity generation will take over as the primary energy source for consumption, to achieve the Millennium Development Goals, the Sustainable Development Goals, and the pledges of the Paris Agreement [1,2]. In fact, electricity, particularly that generated from Renewable Energy Sources (RES), has also been seen as a potential solution to mitigate poverty and the poor's access to energy, mainly experienced in rural areas, and it is hoped that it will promote sustainable growth, and expand economic prospects [1-4]. Therefore, access to affordable and modern energy sources, like electricity produced from RES, could be a vital means for overcoming poverty, and increasing economic growth in a sustainable way [4-6].

To achieve development through an ecological electricity mix, European (EU) countries, have designed and implemented public policies to develop and deploy RES. The installed capacity of RES, and their contribution to energy supply has been growing rapidly [7]. Feed-in Tariffs (FiTs) policies have been largely responsible for the deployment of Intermittent Renewable Energy Sources (RES-I), namely wind power and solar photovoltaic (PV), in EU countries [7]. In fact, the literature argues that environmental concerns, represented by Carbon Dioxide $\left(\mathrm{CO}_{2}\right)$ emissions, and economic wealth, represented by Gross Domestic Product (GDP), have not been effective in promoting RES [8-10]. This research aims to bring fresh new insights about the social and economic drivers of RES deployment, adding the income inequality of households, residential consumption of natural gas, and electricity prices for domestic users in the explanation of RES installed capacity and capacity effectively used, i.e. RES electricity production.

As previously mentioned, RES installed capacity growth has been underpinned by policies to subsidise them. These policies have affected both the economic profits and the economic surplus of economic agents, redistributing the economic surplus between incumbent and incoming electricity generators, and between producers and consumers [11]. So, the installed capacity of RES and its effective use, could actually have been hampering its expected benefits, such as energy security, affordability, and reduced $\mathrm{CO}_{2}$ emissions [12-14]. This research will be focused mainly on the energy affordability problem, because of the literature have warned that RES deployment would increase the cost of electricity for society overall [15-18]. In fact, the literature has studied the affordability problem only to low-income households and solar PV, showing that the higher cost arising from both FiTs and solar PV deployment have threatened low-income households with energy poverty [15-18]. Notwithstanding, the main purpose of this research is to provide and discuss empirical evidence about the effects of all RES deployment on households' income, and on their risk of poverty or social exclusion.

This paper analyses annual data for a panel of fifteen EU countries, from 2005 until 2015. A residual cointegration test was performed to verify the long-run 
relationship between the RES deployment and households living conditions, such as income inequality and risk of poverty or social exclusion. An Autoregressive Distributed Lag (ARDL) methodology was used, because of its ability of dividing the effects into short-run dynamics, and long-run equilibrium. A joint significance test was used to confirm the long-run relationships in the ARDL models. Besides, long-run models were made to prove the robustness of ARDL models long-run results. Therefore, the empirical results allows to answer the following research questions: (i) what is the effect of households' income on RES promotion?; (ii) what are the consequences of promoting renewables on households' income?; and (iii) what is the impact of RES on the risk of poverty or social exclusion of households?

This research provides new evidence and valuable knowledge about the effects of RES on living condition for several types of households, specifically the impact on their income, and their risk of poverty or social exclusion. In short, this research adds new knowledge to the literature, by: (i) the study of the relationships between RES deployment and income inequality; (ii) the analysis of the effects of RES promotion on the risk of poverty or social exclusion; and (iii) the focus on several households types, because of the literature to date has only assessed the effects of RES on low-income. In fact, it is essential to study the effects of RES on all kinds of households, so that policymakers and government fully understand these effects on the economy and on overall society. Therefore, this research aims to fill this gap in the existing literature, as well as to propose and discuss measures by which all consumers could share the economic benefits of the energy transition towards RES.

This paper's results show that solar PV implemented by big players, in the period under analysis, has not yet been beneficial for society. In fact, the solar PV deployed by major producers, has increased the risk of poverty in the overall society. However, the small-scale implementation of solar PV by consumers could bring them into Demand-Side Management (DSM) programs, which could afford them better living conditions, and decrease their risk of poverty. The electrification of home appliances has enhanced the potential for the economic autonomy of households with children, giving them greater security. The results also emphasize that producers have kept all the surpluses from the deployment of RES, and households are threatened with energy poverty. In order to mitigate this negative effect, it is vital that policies are devised to share with consumers the economic surpluses from the deployment of RES. For example, policies could reward consumers with lower electricity prices if they consume in periods with a higher availability of natural resources.

The rest of this paper continues as follows: Section 2 covers the literature on RES drivers, and the consequences of RES implementation on low-income households. The data and methodology are described in Section 3. Section 4 presents and discusses the results. Lastly, Section 5 concludes.

\section{Literature Review}

The literature has being studying the triggers for the deployment of RES. This literature can be divided into two main topics: public policies supporting RES, and economic, environmental, and social drivers of RES implementation [8-10]. The authors of these studies have concluded that fiscal and financial policies have been an effective driver of RES deployment [8-10,19]. In contrast, the empirical literature shows that concerns over the environment, energy dependence, and economic wealth have not been effective drivers of RES deployment [8-10,17]. The literature often shows that the transition to electrical energy from RES has mainly been stimulated by 
two factors: (a) incremental energy consumption; and (b) social and political pressure for the development of cleaner and greener energy sources [8-10,20]. So, the EU countries have replicated the German policy framework for energy transition through the introduction of RES, the Renewable Energy Sources Act (EEG). In fact, German energy policies to promote RES have been the most studied and cited [15-19,21]. However, their results for society and economy have not been those expected or desired, because of their being based on a policy of subsidies, the much-discussed FiTs $[10,15,16,18,22,23]$.

FiTs guarantee dispatch priority, and the production of RES subsidised by FiTs can only be switched off if the grid stability is an inherent concern [24]. All RES generation subsidised by FiTs has a guaranteed price, generally above the market price, which allows high returns for investors [15,19,21,24,25]. Moreover, this policy guarantees all these advantages for producers for at least 20 years, allowing for longterm planning. Electricity utilities that sell electricity directly to consumers have been obligated to accept and pay this fixed price, recovering it through taxes and levies in the electricity price, the so-called RES surcharges. However, these RES surcharges are borne equally by all consumers in their electricity bills, only depending on their consumption. It is true that these policies have enabled less mature and more expensive technologies to be introduced into the market, but at what cost?

In the case of wind power, the wind turbine capacity has increased over time, enabling more electricity to be produced through wind power $[11,25]$. By 2008, EU countries had a significant installed capacity of wind power, providing them a high share of RES in the electricity mix [3,7]. Since then, dispatch priority and a high share of wind power in the electricity mix have increased the percentage of electricity production with a marginal cost close to zero. This led to the so-called merit order effect, putting pressure on, and decreasing the price of electricity generation. This effect has been more noticeable in the wholesale electricity market price $[11,25]$. Nonetheless, the pressure caused by wind power has also had a significant effect on the price of electricity for households and industry. At certain times of the day, indeed, the reduction in the price of electricity has exceeded the additional cost of RES surcharges. So, all electricity consumers have benefited from the deployment of wind power, in contrast to what occurred with the implementation of solar PV $[11,25,26]$.

In the case of solar PV, the author of the first paper of this theme, Frondel et al. [21], argued that solar PV deployment has been an "unfolding disaster", in Germany. In 2015, the same author claimed that solar PV implementation had been a "license to print money" [19]. In fact, the installed capacity of solar PV, in 2007 was almost insignificant. However, by 2015 , it represented a considerable share of electricity production capacity. The capacity of solar PV has grown at a high rate and accumulated high cost, which must continue to be paid for 20 year [19,21]. These cost are mainly due to the high returns provided by FiTs [21]. Consequently, the literature argues that FiTs costs should be diverted to more cost-effective climate protection instruments, diminishing the overall burden of energy transition on the economy $[15,17,18,25]$.

It should be highlighted that FiTs are a technologic-specific policy, and that the FiTs for solar PV guarantee the highest financial support per kilowatt-hour $(\mathrm{kWh})$. This financial support has been set, because solar PV modules are still inefficient, and geographical conditions are mostly unfavourable for them in EU countries outside the Iberian Peninsula [17]. The policy was implemented to offset the lack of competitiveness of this particular RES technology [26]. However, the literature has argued that the effect of incentivizing solar PV through FiTs has been harmful to households [15-18]. In fact, solar PV deployment has greatly increased households' 
electricity bills. Thus, the literature has been studying this effect, and how to mitigate it, particularly for low-income households [15-18].

The literature has only assessed the effects of PV implementation for two countries, namely Germany and Australia, by major players in solar PV [15-18]. The authors of these studies noted that wealthy households have the opportunity to invest, indirectly through savings, or directly through the installation of PV panels in their homes [15-18]. However, neither of the papers studied the direct impact of solar PV on wealthy households' income or budgets, and the impact of wealthy households on solar PV deployment. In assessing the impact of RES deployment through the price of electricity, studies have only focused on low-income households. This literature has pointed out that poorer households have financed the substantial cost of RES deployment, through their electricity bills [15-18]. As the RES surcharges in electricity bills is proportional to the electricity consumed, both wealthy and poorer households pay the same surcharge [15-18]. This redistribution of costs through surcharges, has led the authors to argue that the burden of RES implementation is higher for poor households than for wealthy households [15-19,21]. Thus, the literature has been researching and discussing policies and measures for energy transition that do not threaten poorer households with energy poverty [15-19,21].

The shift from fossil fuels to RES in electricity generation systems, has virtually doubled the price of electricity, with this increase being split between the cost of generation and RES surcharges, since the introduction of FiTs policies [15-19,21]. Studies of deploying wind power have only analysed its effect on the wholesale electricity market price [11,25]. The effect of solar PV deployment through FiTs schemes has only been assessed for low-income households in Germany and Australia. However, it is crucial to analyse the effects of RES, disaggregated by source, on all household types. In fact, it is expected that the energy transition from fossil fuels to RES in the electricity generation, and the transition from fossil fuels towards electricity in households consumption will have consequences on the overall society. This theme has not experienced much of the attention by the literature and scientific community. However, it is pertinent to understand the benefits and disadvantages that will be provoked in the households. Has the transition benefited the overall society, or it has benefited one type of households while harming other types? Therefore, it is crucial to realize the effects of the measures to deal effectively with global warming, and more important, if these measures could constitute an exclusion of most people from the new energies and their benefits. Energy policy makers should be aware of the benefits and potential disadvantages for society, protecting the low-income households through a fair redistributing of the benefits of RES production and electricity consumption between the high- and low-income households.

\section{Data and Methodology}

To accomplish the objectives of this study, the database of the European Union Statistics on Income and Living Conditions (EU-SILC) was used. This database allows the income and poverty of households to be analysed through comparable crosssectional, and longitudinal multidimensional data. This research also used other drivers of income inequality in populations, such as work intensity, education levels, and natural gas consumption; and drivers of RES implementation in economies, namely gross domestic product, electricity prices, and energy intensity. The selection of EU countries was made according to the following requisites: (i) data on the installed 
capacities of wind power, solar PV, and hydro power being available and higher than zero; and (ii) accessible data about the income and risk of poverty of all types of household for the entire time-span without gaps, which the EU-SILC database contains. So, this analysis focuses on the following fifteen countries: Austria, Belgium, Czech Republic, Denmark, Finland, France, Germany, Greece, Italy, Luxembourg, the Netherlands, Portugal, Spain, Sweden and the United Kingdom. Annual data was used for a time-span from 2005 until 2015.

The mean net income, the people at risk of poverty and social exclusion, and the percentage of people with very low work intensity was retrieved from the EU-SILC database by household type. The mean net income variable is the mean monetary value of wages, rents, and interests earned by each household type. The people at risk of poverty or social exclusion variable indicates the sum of persons who are at risk of poverty or being severely materially deprived or living in households with very low work intensity. This variable accounts for persons with an equivalised disposable income below the risk-of-poverty threshold, which is set at $60 \%$ of the national median disposable income. The people with very low work intensity variable designates the percentage of people living in households where the adults work $20 \%$ or less of their total work potential. Table A.1 in the appendix, shows the distribution of population by percentage of household type. The installed capacity of wind power, solar PV, and hydropower; the share of electricity produced through RES; the price of electricity for households; natural gas consumption in residences; gross domestic product; and the percentage of government expenditure on education, have all been retrieved from the Eurostat database. It should be noted that the electricity variables are only related to major producers and auto-producers, because of the non-availability of statistics on prosumers.

Table 1. Descriptive statistics of variables

\begin{tabular}{|c|c|c|c|c|c|}
\hline Variable & Obs & Mean & Std. Dev. & Min & Max \\
\hline \multicolumn{6}{|c|}{ Percentage of household's mean disposable income in relation to the total mean disposable income (\%) } \\
\hline Single person & 165 & 87.76 & 7.14 & 75.04 & 106.59 \\
\hline Single person with dependent children & 165 & 71.23 & 5.32 & 58.72 & 88.32 \\
\hline Two adults & 165 & 111.05 & 5.15 & 96.61 & 123.27 \\
\hline Two adults younger than 65 years & 165 & 122.29 & 4.74 & 111.51 & 133.73 \\
\hline Two adults, at least one aged 65 years or over & 165 & 96.94 & 8.64 & 79.31 & 119.18 \\
\hline Two adults with one dependent child & 165 & 108.51 & 4.70 & 99.92 & 124.00 \\
\hline Two adults with two dependent children & 165 & 100.31 & 5.27 & 89.21 & 115.14 \\
\hline Two adults with three or more dependent children & 165 & 84.95 & 6.61 & 70.00 & 108.52 \\
\hline Two adults or more without dependent children & 165 & 111.83 & 3.54 & 101.90 & 122.54 \\
\hline Two adults or more with dependent children & 165 & 98.24 & 3.95 & 90.95 & 108.09 \\
\hline Three or more adults & 165 & 113.24 & 5.05 & 99.93 & 130.24 \\
\hline Three or more adults with dependent children & 165 & 92.20 & 7.95 & 73.15 & 123.29 \\
\hline Households without dependent children & 165 & 104.63 & 3.42 & 97.33 & 114.14 \\
\hline Households with dependent children & 165 & 95.56 & 3.15 & 89.46 & 102.96 \\
\hline \multicolumn{6}{|c|}{ Number of people at risk of poverty or social exclusion (number of people) } \\
\hline Single person & 165 & 32.16 & 5.58 & 16.70 & 47.00 \\
\hline Single person with dependent children & 165 & 46.46 & 7.92 & 28.80 & 74.70 \\
\hline Two adults & 165 & 16.65 & 6.51 & 7.90 & 36.60 \\
\hline Two adults younger than 65 years & 165 & 17.51 & 6.08 & 9.50 & 36.40 \\
\hline Two adults, at least one aged 65 years or over & 165 & 15.64 & 8.22 & 4.60 & 40.40 \\
\hline Two adults with one dependent child & 165 & 14.85 & 5.85 & 5.00 & 37.50 \\
\hline Two adults with two dependent children & 165 & 14.86 & 7.40 & 4.40 & 33.90 \\
\hline Two adults with three or more dependent children & 165 & 27.50 & 10.73 & 9.80 & 51.20 \\
\hline Two adults or more without dependent children & 165 & 15.73 & 5.90 & 8.00 & 33.50 \\
\hline Two adults or more with dependent children & 165 & 17.78 & 7.21 & 7.80 & 38.30 \\
\hline Three or more adults & 165 & 14.08 & 6.41 & 3.90 & 39.00 \\
\hline Three or more adults with dependent children & 165 & 20.32 & 9.77 & 1.50 & 50.90 \\
\hline Households without dependent children & 165 & 20.69 & 4.53 & 11.70 & 34.10 \\
\hline Households with dependent children & 165 & 20.59 & 6.45 & 11.10 & 38.80 \\
\hline
\end{tabular}




\begin{tabular}{|c|c|c|c|c|c|}
\hline Total households & 165 & 9.60 & 2.79 & 4.70 & 18.10 \\
\hline Single person & 165 & 22.26 & 6.00 & 9.90 & 35.10 \\
\hline Single person with dependent children & 165 & 26.11 & 8.77 & 11.00 & 50.40 \\
\hline Two adults & 165 & 12.88 & 4.55 & 4.30 & 25.30 \\
\hline Two adults younger than 65 years & 165 & 10.87 & 3.45 & 3.90 & 20.20 \\
\hline Two adults, at least one aged 65 years or over & 165 & 36.21 & 8.96 & 8.90 & 56.70 \\
\hline Two adults with one dependent child & 165 & 5.17 & 1.92 & 1.70 & 11.70 \\
\hline Two adults with two dependent children & 165 & 3.33 & 1.78 & 0.50 & 9.40 \\
\hline Two adults with three or more dependent children & 165 & 6.86 & 4.03 & 0.00 & 19.20 \\
\hline Two adults or more without dependent children & 165 & 10.97 & 4.06 & 4.40 & 26.90 \\
\hline Two adults or more with dependent children & 165 & 4.92 & 2.32 & 1.90 & 13.40 \\
\hline Three or more adults & 165 & 8.42 & 4.85 & 2.80 & 28.10 \\
\hline Three or more adults with dependent children & 165 & 6.15 & 4.62 & 0.10 & 23.40 \\
\hline Households without dependent children & 165 & 14.25 & 3.80 & 8.30 & 26.70 \\
\hline Households with dependent children & 165 & 7.01 & 2.76 & 2.90 & 14.50 \\
\hline
\end{tabular}

The mean net income by household has been divided by the total mean income, and multiplied by 100, to show the percentage of household income in relation to the total disposable income. This variable is able to measure income inequality by household, so it can be discovered if the income of a household is above or below the mean income. This variable neglects other economic effects, such as any increase in the national base salary, but this is not relevant to this analysis. Subsequently, all series were transformed into their natural logarithms, denoted by the prefix " $L$ ". Table 1 summarises the descriptive statistics of the EU-SILC data, and Table 2 shows the descriptive statistics of energy, social, and economic series.

Table 2. Descriptive statistics of energy and control variables

\begin{tabular}{|c|c|c|c|c|c|c|}
\hline Variable & Definition and measure & Obs & Mean & Std. Dev. & Min & Max \\
\hline$R E S \_I C$ & $\begin{array}{c}\text { Installed capacity of renewable } \\
\text { energies (MW) }\end{array}$ & 165 & 16662.19 & 18685.20 & 1193 & 95883 \\
\hline HYDRO_IC & $\begin{array}{c}\text { Installed capacity of hydro power } \\
\text { (MW) }\end{array}$ & 165 & 8444.56 & 8245.70 & 7 & 25401 \\
\hline WIND_IC & $\begin{array}{l}\text { Installed capacity of wind power } \\
\text { (MW) }\end{array}$ & 165 & 5433.82 & 8225.61 & 22 & 44670 \\
\hline$S O L \_I C$ & Installed capacity of solar (MW) & 165 & 2717.82 & 6737.86 & 1 & 39788 \\
\hline RES_GEN & $\begin{array}{c}\text { Electricity production from renewable } \\
\text { energies }(1000 \mathrm{TOE})\end{array}$ & 165 & 9212.81 & 8235.37 & 71.60 & 38886.10 \\
\hline HYDRO_GEN & $\begin{array}{l}\text { Electricity production from hydro } \\
\text { power (1000 TOE) }\end{array}$ & 165 & 1696.80 & 1926.42 & 1.10 & 6786.90 \\
\hline WIND_GEN & $\begin{array}{l}\text { Electricity production from wind } \\
\text { power (1000 TOE) }\end{array}$ & 165 & 893.69 & 1273.60 & 1.80 & 6810.50 \\
\hline SOL_GEN & $\begin{array}{l}\text { Electricity production from solar } \\
\text { (1000 TOE) }\end{array}$ & 165 & 360.74 & 763.73 & 0.70 & 4001.10 \\
\hline PRICE ELEC & Electricity price (euro per KWh) & 165 & 0.18 & 0.05 & 0.07 & 0.31 \\
\hline GAS_CONS & Natural gas consumption in residences & 165 & 6491.90 & 8575.19 & 26.30 & 30149.30 \\
\hline GEH_INTS & $\begin{array}{c}\text { Greenhouse gas emissions intensity of } \\
\text { energy consumption (index) }\end{array}$ & 165 & 92.33 & 6.86 & 74.20 & 108.90 \\
\hline ENERG_INTS & $\begin{array}{l}\text { Energy intensity of the economy (kg } \\
\text { of oil equivalent per } 1000 \text { EUR) }\end{array}$ & 165 & 137.30 & 46.99 & 65.10 & 327 \\
\hline$G D P$ & $\begin{array}{l}\text { Gross domestic product per capita } \\
\text { (constant LCU) }\end{array}$ & 165 & 99185.23 & 133694.90 & 16028.16 & 405353.30 \\
\hline$E D U_{-} E X P S$ & $\begin{array}{c}\text { Percentage of government expenditure } \\
\text { in education }\end{array}$ & 165 & 11.09 & 1.78 & 7.30 & 15.10 \\
\hline
\end{tabular}

Note: TOE means tonnes of oil equivalent; KWh mean Kilowatt hour; LCU means local currency unit

The results of the CD-test proposed by Pesaran [27], the CD-test is the most employed and accurate to test the cross-section dependence by variable [27-29]. The CD-test supports the presence of cross-sectional dependence in most series (see Table 3 ). However, in the hydro power series and income series, the CD-test does not support the presence of cross-sectional dependence. As a consequence of the CD-test results, 
both the first- and second-generation unit roots tests were performed. Only the secondgeneration tests are displayed (see Table 3), because of the conformity between the first ${ }^{2}$ and second-generation tests. The results of the CIPS test proposed by Pesaran [27], were robust in the presence of cross-sectional dependence, and revealed that all series are integrated of order one, i.e. I(1).

Table 3. Cross section dependence and unit roots test

\begin{tabular}{|c|c|c|c|c|c|c|c|}
\hline & \multicolumn{3}{|c|}{ Cross section dependence } & \multicolumn{4}{|c|}{ Unit roots test (CIPS) } \\
\hline & \multirow[b]{2}{*}{ CD-test } & \multirow[b]{2}{*}{ Corr } & \multirow[b]{2}{*}{$\begin{array}{c}\text { Abs } \\
\text { (Corr) }\end{array}$} & \multicolumn{2}{|c|}{ Level } & \multicolumn{2}{|c|}{ First differences } \\
\hline & & & & $\begin{array}{c}\text { No } \\
\text { trend }\end{array}$ & Trend & $\begin{array}{c}\text { No } \\
\text { trend }\end{array}$ & Trend \\
\hline \multicolumn{8}{|c|}{ Percentage of household's mean disposable income in relation to the total mean disposable income (in natural logarithm) } \\
\hline Single person & 0.21 & 0.006 & 0.42 & -0.661 & 1.087 & $-5.538^{* * *}$ & $-3.474 * * *$ \\
\hline Single person with dependent children & -0.57 & -0.017 & 0.291 & -0.531 & -0.552 & $-5.309^{* * *}$ & $-1.762 * *$ \\
\hline Two adults & $3.92 * * *$ & 0.115 & 0.415 & -1.244 & 1.153 & $-4.358 * * *$ & $-2.616^{* * *}$ \\
\hline Two adults younger than 65 years & 1.39 & 0.041 & 0.319 & 0.215 & 1.151 & $-3.441^{* * *}$ & $-1.929 * *$ \\
\hline Two adults, at least one aged 65 years or over & $19.79 * * *$ & 0.582 & 0.601 & 15.421 & 13.817 & $-4.960 * * *$ & $-2.902 * * *$ \\
\hline Two adults with one dependent child & -0.71 & -0.021 & 0.258 & 0.618 & -0.09 & $-4.318 * * *$ & $-1.663 * *$ \\
\hline Two adults with two dependent children & $2.19 * *$ & 0.064 & 0.274 & -0.12 & 1.806 & $-3.087 * * *$ & $-2.080 * *$ \\
\hline Two adults with three or more dependent children & 0.74 & 0.022 & 0.37 & -0.699 & 1.803 & $-6.916^{* * *}$ & $-4.310^{* * *}$ \\
\hline Two adults or more without dependent children & $2.29 * *$ & 0.067 & 0.342 & -0.88 & -0.579 & $-4.196^{* * *}$ & $-2.585 * * *$ \\
\hline Two adults or more with dependent children & 0.64 & 0.019 & 0.407 & -0.613 & -0.723 & $-4.157 * * *$ & $-2.187 * *$ \\
\hline Three or more adults & $2.48^{* *}$ & 0.073 & 0.286 & 15.421 & 13.817 & $-6.228 * * *$ & $-3.429 * * *$ \\
\hline Three or more adults with dependent children & 1.54 & 0.045 & 0.321 & -0.136 & -0.037 & $-5.655^{* * *}$ & $-3.793 * * *$ \\
\hline Households without dependent children & -0.06 & -0.002 & 0.419 & -0.553 & 0.117 & $-3.478 * * *$ & $-1.560 * *$ \\
\hline Households with dependent children & -0.16 & -0.005 & 0.423 & -0.796 & 0.015 & $-3.812 * * *$ & $-1.876^{* *}$ \\
\hline \multicolumn{8}{|c|}{ Number of people at risk of poverty or social exclusion (in natural logarithm) } \\
\hline Single person & -0.43 & -0.013 & 0.406 & -0.804 & -0.21 & $-4.116^{* * *}$ & $-1.886^{* *}$ \\
\hline Single person with dependent children & -1.59 & -0.047 & 0.285 & 0.423 & -0.017 & $-5.191 * * *$ & $-4.293 * * *$ \\
\hline Two adults & $11.62 * * *$ & 0.342 & 0.425 & -1.261 & -0.876 & $-3.651^{* * *}$ & $-1.911^{* *}$ \\
\hline Two adults younger than 65 years & 0.5 & 0.015 & 0.4 & -0.287 & -1.245 & $-3.890^{* * *}$ & $-2.060 * *$ \\
\hline Two adults, at least one aged 65 years or over & $14.19 * * *$ & 0.418 & 0.561 & 15.421 & -0.469 & $-3.947 * * *$ & $-1.669 * *$ \\
\hline Two adults with one dependent child & $5.27 * * *$ & 0.155 & 0.332 & 0.101 & 0.945 & $-4.551 * * *$ & $-1.644 * *$ \\
\hline Two adults with two dependent children & $3.05 * * *$ & 0.09 & 0.252 & -0.676 & -1.042 & $-4.481 * * *$ & $-2.169^{* *}$ \\
\hline Two adults with three or more dependent children & 0.05 & 0.002 & 0.274 & 1.137 & 2.708 & $-5.178^{* * *}$ & $-2.770 * * *$ \\
\hline Two adults or more without dependent children & $2.68 * * *$ & 0.079 & 0.347 & 0.398 & -1.113 & $-4.855^{* * *}$ & $-2.489 * * *$ \\
\hline Two adults or more with dependent children & $6.88^{* * *}$ & 0.202 & 0.361 & 15.421 & 13.817 & $-4.486 * * *$ & $-1.739 * *$ \\
\hline Three or more adults & $6.86^{* * *}$ & 0.202 & 0.329 & 15.421 & 13.817 & $-5.300 * * *$ & $-2.684 * * *$ \\
\hline Three or more adults with dependent children & $2.68 * * *$ & 0.079 & 0.368 & -1.526 & 1.22 & $-6.181 * * *$ & $-3.638 * * *$ \\
\hline Households without dependent children & 0.65 & 0.019 & 0.37 & 0.375 & 13.817 & $-4.682 * * *$ & $-2.591 * * *$ \\
\hline Households with dependent children & $6.05^{* * *}$ & 0.178 & 0.384 & 15.421 & -2.772 & $-5.108 * * *$ & $-2.133 * *$ \\
\hline \multicolumn{8}{|c|}{ Percentage of people living with very low work intensity (in natural logarithm) } \\
\hline Total households & $8.48^{* * *}$ & 0.25 & 0.41 & & & $* *$ & ** \\
\hline Single person & $7.81 * * *$ & 0.23 & 0.416 & -1.008 & -0.761 & $-4.969 * * *$ & $-2.363 * * *$ \\
\hline Single person with dependent children & 0.98 & 0.029 & 0.323 & 15.421 & -0.667 & $-5.637 * * *$ & $-3.467 * * *$ \\
\hline Two adults & $2.73 * * *$ & 0.08 & 0.487 & 0.156 & -0.105 & $-3.116^{* * *}$ & $-1.637 * *$ \\
\hline Two adults younger than 65 years & $3.43^{* * *}$ & 0.101 & 0.481 & 0.545 & -0.27 & $-3.312 * * *$ & $-1.191 * *$ \\
\hline Two adults, at least one aged 65 years or over & -0.43 & -0.013 & 0.401 & -0.771 & -0.789 & $-4.301 * * *$ & $-1.670^{* *}$ \\
\hline Two adults with one dependent child & $5.01 * * *$ & 0.147 & 0.316 & 0.588 & -0.402 & $-3.584 * * *$ & $-1.650^{* *}$ \\
\hline Two adults with two dependent children & $2.32 * * *$ & 0.068 & 0.331 & -1.101 & -0.177 & $-5.394 * * *$ & $-3.656 * * *$ \\
\hline Two adults with three or more dependent children & $2.04 * *$ & 0.06 & 0.291 & 1.435 & 3.251 & $-5.194 * * *$ & $-2.717 * * *$ \\
\hline Two adults or more without dependent children & $2.51 * * *$ & 0.074 & 0.404 & -0.335 & 0.33 & $-5.347 * * *$ & $-3.733 * * *$ \\
\hline Two adults or more with dependent children & $6.59 * * *$ & 0.194 & 0.366 & 0.424 & 0.246 & $-6.960 * * *$ & $-4.775 * * *$ \\
\hline Three or more adults & $4.32 * * *$ & 0.127 & 0.32 & 0.588 & -0.753 & $-5.859 * * *$ & $-3.698 * * *$ \\
\hline Three or more adults with dependent children & $2.54 * *$ & 0.075 & 0.355 & -0.911 & -0.684 & $-6.102 * * *$ & $-3.062 * * *$ \\
\hline Households without dependent children & $6.99 * * *$ & 0.206 & 0.406 & 15.421 & 13.817 & $-6.692 * * *$ & $-4.143 * * *$ \\
\hline Households with dependent children & $7.91 * * *$ & 0.233 & 0.377 & -0.76 & 1.083 & $-6.041 * * *$ & $-3.529 * * *$ \\
\hline \multicolumn{8}{|l|}{ Energy and control variables } \\
\hline LRES_IC & $31.53 * * *$ & 0.928 & 0.928 & 0.371 & 0.957 & $-1.497 * * *$ & $-2.036^{* *}$ \\
\hline LHYDRO IC & - & - & - & 2.174 & 3.857 & $-0.590 * *$ & $-0.439 * * *$ \\
\hline LWIND $\overline{I C}$ & $31.19 * * *$ & 0.918 & 0.918 & -0.599 & 1.599 & $-1.745 * * *$ & $-1.877 * * *$ \\
\hline$L S O L \_I C$ & $30.33 * * *$ & 0.892 & 0.892 & 0.072 & 1.623 & $-1.408 * *$ & $-0.072 * * *$ \\
\hline LRES_GEN & $29.23 * * *$ & 0.866 & 0.866 & -0.988 & 0.271 & $-3.077 * * *$ & $-0.989 * *$ \\
\hline LHYDRO_GEN & $3.76^{* * *}$ & 0.111 & 0.354 & -0.394 & 0.147 & $-4.228 * * *$ & $-1.695 * *$ \\
\hline LWIND_GEN & $30.42 * * *$ & 0.9 & 0.9 & -0.551 & 0.638 & $-2.143 * *$ & $-0.960 * *$ \\
\hline$L S O L \bar{G} E N$ & $31.89 * * *$ & 0.944 & 0.944 & 15.155 & -0.768 & $-2.689 * * *$ & $-1.879 * * *$ \\
\hline$L P R I \bar{C} E \_E L E C$ & $21.05 * * *$ & 0.619 & 0.725 & -0.222 & -1.135 & $-4.383 * * *$ & $-3.884 * * *$ \\
\hline
\end{tabular}

2 They are not presented, but are available upon request to the authors. 


\begin{tabular}{|c|c|c|c|c|c|c|c|}
\hline$L G A S C O N S$ & $13.34 * * *$ & 0.393 & 0.512 & -0.771 & 1.008 & $-1.599 * * *$ & $-9.537 * * *$ \\
\hline LGEH_INTS & $18.23 * * *$ & 0.536 & 0.662 & -1.002 & 1.241 & $-1.978 * * *$ & $-1.778 * *$ \\
\hline LENER $G$ INTS & $24.97 * * *$ & 0.735 & 0.78 & -0.8 & -0.105 & $-2.693^{* * *}$ & $-1.688 * * *$ \\
\hline$L G D P$ & $12.96^{* * *}$ & 0.381 & 0.513 & 3.417 & 1.64 & $-1.099 * * *$ & $1.752 * * *$ \\
\hline$L E D U$ EXPS & $1.08 * * *$ & 0.032 & 0.446 & 0.409 & 0.421 & $-4.567 * * *$ & $-3.052 * * *$ \\
\hline
\end{tabular}

Notes: **,***, denote statistical significance at $5 \%$ and $1 \%$ level, respectively. CD-test has $\mathrm{N}(0,1)$ distribution under $\mathrm{H} 0$ : cross- section independence; panel unit roots test (CIPS) tests the H0: series are I(1).

To show the effects of household income and the usual RES drivers [9,20,30,31] on the deployment and use of RES, eight models were estimated, namely (with their respective dependent variable):

- WIND_IC - installed capacity of wind power;

- $S O L \_-\overline{I C}$ - installed capacity of solar PV;

- HYDRO_IC - installed capacity of hydro power;

- RES_IC-installed capacity of all RES;

- WIND_GEG - electricity generation from wind power;

- SOL_GEG - electricity generation from solar PV;

- HYDRO_GEG - electricity generation from hydro power; and

- RES_GEG - electricity generation from all RES.

To meet the main objective of this paper, 14 models were estimated to reveal the effects of the deployment of RES on household incomes, and also to reveal the effects on the households' risk of poverty and social exclusion, using as dependent variables the household' income and the risk of poverty, respectively. This research also tested the drivers of income inequality generally used in the literature [32-35].

Table 4. Kao residual cointegration test

\begin{tabular}{|c|c|c|c|c|c|}
\hline Single person & $\begin{array}{l}\text { inc } \\
\text { risk }\end{array}$ & $\begin{array}{l}-5.201271 * * * \\
-3.814086^{* * *}\end{array}$ & $\begin{array}{l}\text { Two or more adults with } \\
\text { dependent children }\end{array}$ & $\begin{array}{l}\text { inc } \\
\text { risk }\end{array}$ & $\begin{array}{l}-4.880614 * * * \\
-7.910950 * * *\end{array}$ \\
\hline \multirow{2}{*}{$\begin{array}{l}\text { Single person with } \\
\text { dependent children }\end{array}$} & & $-3.513028 * * *$ & \multirow{2}{*}{ Three or more adults } & $i n c$ & $-4.799234 * * *$ \\
\hline & risk & $-7.464221 * * *$ & & risk & $-7.586330 * * *$ \\
\hline \multirow{2}{*}{ Two adults } & inc & $-3.245477 * * *$ & \multirow{2}{*}{$\begin{array}{l}\text { Three or more adults with } \\
\text { dependent children }\end{array}$} & inc & $-3.792484 * * *$ \\
\hline & risk & $-3.757293 * * *$ & & risk & $-3.490349 * * *$ \\
\hline \multirow{2}{*}{$\begin{array}{l}\text { Two adults younger than } \\
65 \text { years }\end{array}$} & inc & $-5.837310 * * *$ & \multirow{2}{*}{$\begin{array}{l}\text { Households without } \\
\text { dependent children }\end{array}$} & inc & $-4.080159 * * *$ \\
\hline & risk & $-7.638724 * * *$ & & risk & $-3.930485 * * *$ \\
\hline \multirow{2}{*}{$\begin{array}{l}\text { Two adults, at least one } \\
\text { aged } 65 \text { years or over }\end{array}$} & inc & $-4.939195 * * *$ & \multirow{2}{*}{$\begin{array}{l}\text { Households with } \\
\text { dependent children }\end{array}$} & inc & $-4.410320 * * *$ \\
\hline & risk & $-4.153939 * * *$ & & risk & $-7.640866 * * *$ \\
\hline \multirow{2}{*}{$\begin{array}{l}\text { Two adults with one } \\
\text { dependent child }\end{array}$} & inc & $-5.592643 * * *$ & RES_IC & & $-2.117332 * * *$ \\
\hline & risk & $-6.265151 * * *$ & RES_GEN & & $-3.854390 * * *$ \\
\hline \multirow{2}{*}{$\begin{array}{l}\text { Two adults with two } \\
\text { dependent children }\end{array}$} & inc & $-2.810666^{* * *}$ & HYDRO_IC & & $-5.354045 * * *$ \\
\hline & risk & $-8.446245^{* * *}$ & HYDRO_GEN & & $-3.691919 * * *$ \\
\hline \multirow{2}{*}{$\begin{array}{l}\text { Two adults with three or } \\
\text { more dependent children }\end{array}$} & inc & $-4.091316^{* * *}$ & WIND_IC & & $-5.343441 * * *$ \\
\hline & risk & $-7.126844 * * *$ & WIND_GEN & & $-4.871691 * * *$ \\
\hline \multirow{2}{*}{$\begin{array}{l}\text { Two or more adults } \\
\text { without dependent } \\
\text { children }\end{array}$} & inc & $-3.580205 * * *$ & $S O L_{-} I C$ & & $-5.120046^{* * *}$ \\
\hline & risk & $-4.891898 * * *$ & SOL_GEN & & $-4.276471 * * *$ \\
\hline
\end{tabular}

Notes: $* * *$ denote statistical significance at $1 \%$ level; inc refers to the percentage of household's mean disposable income in relation to the total mean disposable income, and risk refers to the number of people at risk of poverty or social exclusion. 
As all variables are I(1), the Kao residual cointegration test [36] was employed. This test is based on a Monte Carlo procedure, which outperforms the Pedroni's test when there is a small time series dimension in panel data [36], as is the case of this analysis. The Kao tests suggest the existence of long-run relationships in all models. Thus, the analysis of both short-run adjustments and long-run equilibrium are recommended. An ARDL methodology has been applied to apportion the total effects into short- and long-runs. Furthermore, the ARDL methodology allows the use of I(1) variables, and is suitable for long memory patterns. The literature suggests that ARDL models applied in panel data produce consistent and efficient parameter estimations, even in small-samples [37,38]. The ARDL equation modelling and estimates the shortand long-run coefficients simultaneously, eliminating econometric problems associated with omitted variables and autocorrelation. The coefficients estimation provided by the ARDL equation, which is a cointegration method, are unbiased and efficient, mainly because of its avoidance of problems that might happen in the presence of serial correlation and endogeneity [39-42]. Moreover, traditional estimators, even traditional cointegration estimators, may produce erroneous results due to the endogeneity, meanwhile, with the ARDL method the independent and dependent variables could be distinguished and produced efficient results under endogeneity issues [38-43]. Therefore, the general ARDL models used are stated below (eq. 1). To prove the existence of long-run relationships in the ARDL models, two Joint Significance Tests (JSE) were carried out, using a Wald test, an individual test (eq. 2) and a joint test (eq. $3)$.

$$
\begin{aligned}
D \boldsymbol{Y}_{i, t}= & f\left(D \boldsymbol{X}_{i, t}, \boldsymbol{Y}_{i, t-1}, \boldsymbol{X}_{i, t-1}\right) \\
& \mathrm{H} 0: \alpha_{\mathrm{i}}=\alpha \mathrm{i}+1=0 \\
& \mathrm{H} 0: \alpha_{\mathrm{i}}+\alpha_{\mathrm{i}}+1=0,
\end{aligned}
$$

where, $\mathbf{Y}_{\mathrm{i}, \mathrm{t}}$ is the vector of dependent variables, $\mathbf{X}_{\mathrm{i}, \mathrm{t}}$ is the vector of independent variables, and the $\alpha_{i}$ denotes the significant long-run coefficients in the models. The variables incorporated in the models are in natural logarithms, and first differences of logarithms, denoted by the operator "D", their coefficients are elasticities (long-run) and semi-elasticities (short-run). The elasticities are computed by dividing the coefficient of the long-run series by the coefficient of the Error Correction Mechanism (ECM), from the estimated models both lagged once, and then multiplied by -1 .

The correlation matrix and the variance inflation factor statistics revealed that the income percentage of some households had to be excluded from the RES models, because of problems of collinearity and multicollinearity. Thereafter, the low correlation values and VIF statistics support the idea that collinearity and multicollinearity was no longer a concern. A battery of model specification tests were performed to show the properties of the models (see Table 5).

Table 5. Model specification tests

\begin{tabular}{lllllll}
\hline Models & $\begin{array}{l}\text { Hausman } \\
\text { RE vs. FE }\end{array}$ & $\begin{array}{l}\text { Modified Wald } \\
\text { test }\end{array}$ & $\begin{array}{l}\text { Wooldridge } \\
\text { test }\end{array}$ & $\begin{array}{l}\text { Pesaran's } \\
\text { test }\end{array}$ & $\begin{array}{l}\text { Frees' } \\
\text { test }\end{array}$ & $\begin{array}{l}\text { Friedman's } \\
\text { test }\end{array}$ \\
\hline$R E S I C$ & $35.87^{* * *}$ & $809.92^{* * *}$ & $21.406^{* * *}$ & -0.36 & $0.626^{* * *}$ & 5.953 \\
\hline$R E S G E N$ & $46.23^{* * *}$ & $234.68^{* * *}$ & $7.283^{* *}$ & 0.575 & -0.191 & 14.36 \\
\hline$H Y D R O I C$ & $53.05^{* * *}$ & $609.94^{* * *}$ & $75.659^{* * *}$ & 0.908 & -0.097 & 13.022 \\
\hline$H Y D R O G E N$ & $73.04^{* * *}$ & $493.64^{* * *}$ & $66.014^{* * *}$ & 1.304 & $0.498^{* *}$ & 13.691 \\
\hdashline$W I N D I C$ & $70.65^{* * *}$ & $132.92^{* * *}$ & $9.555^{* * *}$ & -0.548 & 0.13 & 6.142 \\
\hline$W I N D G E N$ & $39.41^{* * *}$ & $49.37^{* * *}$ & 0.803 & $1.811^{* *}$ & 0.183 & 18.491 \\
\hline$S O L I C$ & $41.69^{* * *}$ & $585.79^{* * *}$ & $18.796^{* * *}$ & -0.394 & $0.257^{*}$ & 7.393 \\
\hline$S O L G E N$ & $50.63^{* * *}$ & $62.99^{* * *}$ & $39.344^{* * *}$ & -0.369 & $0.697^{* * *}$ & 6.578 \\
\hline
\end{tabular}




\begin{tabular}{|c|c|c|c|c|c|c|c|}
\hline Single person & $\begin{array}{l}\text { inc } \\
\text { risk }\end{array}$ & $\begin{array}{l}47.80 * * * \\
44.54 * * *\end{array}$ & $\begin{array}{l}115.75 * * * \\
107.69 * * *\end{array}$ & $\begin{array}{l}23.058 * * * \\
26.516 * * *\end{array}$ & $\begin{array}{l}-1.213 \\
-1.275\end{array}$ & $\begin{array}{l}0.213 \\
-0.258\end{array}$ & $\begin{array}{l}4.135 \\
5.415\end{array}$ \\
\hline \multirow{2}{*}{$\begin{array}{l}\text { Single person with } \\
\text { dependent children }\end{array}$} & $i n c$ & $42.53 * * *$ & $96.82 * * *$ & $26.520 * * *$ & 0.709 & 0.159 & 8.76 \\
\hline & risk & $61.32 * * *$ & $209.07 * * *$ & $17.624 * * *$ & -0.892 & $0.594 * * *$ & 4.978 \\
\hline \multirow{2}{*}{ Two adults } & $i n c$ & $30.25 * * *$ & $116.16^{* * *}$ & $26.180 * * *$ & -0.557 & -0.363 & 11.349 \\
\hline & risk & $39.03 * * *$ & $251.33 * * *$ & $23.343 * * *$ & -1.006 & 0.122 & 4.847 \\
\hline \multirow{2}{*}{$\begin{array}{l}\text { Two adults } \\
\text { younger than } 65 \\
\text { years }\end{array}$} & $i n c$ & $33.62 * * *$ & $255.18 * * *$ & $43.448 * * *$ & 1.001 & 0.137 & 12.862 \\
\hline & risk & $56.10 * * *$ & $63.80 * * *$ & $83.311 * * *$ & -0.935 & 0.029 & 5.327 \\
\hline \multirow{2}{*}{$\begin{array}{l}\text { Two adults, at least } \\
\text { one aged } 65 \text { years } \\
\text { or over }\end{array}$} & inc & $42.72 * * *$ & $83.88^{* * *}$ & $56.349 * * *$ & -0.747 & -0.226 & 7.873 \\
\hline & risk & $38.07 * * *$ & $207.26 * * *$ & $22.498 * * *$ & -1.085 & 0.09 & 5.007 \\
\hline \multirow{2}{*}{$\begin{array}{l}\text { Two adults with } \\
\text { one dependent } \\
\text { child }\end{array}$} & $i n c$ & $50.47 * * *$ & $213.47 * * *$ & $24.413 * * *$ & -1.568 & $0.272 *$ & 2.476 \\
\hline & risk & $54.52 * * *$ & $158.16 * * *$ & $48.203 * * *$ & -0.566 & $0.308^{*}$ & 5.255 \\
\hline \multirow{2}{*}{$\begin{array}{l}\text { Two adults with } \\
\text { two dependent } \\
\text { children }\end{array}$} & inc & $47.39 * * *$ & $136.61 * * *$ & $18.947 * * *$ & -0.365 & -0.072 & 9.153 \\
\hline & risk & $75.26 * * *$ & $138.48 * * *$ & $9.679 * * *$ & 0.204 & -0.201 & 5.371 \\
\hline \multirow{2}{*}{$\begin{array}{l}\text { Two adults with } \\
\text { three or more } \\
\text { dependent children }\end{array}$} & inc & $43.12^{* * *}$ & $261.95 * * *$ & $31.386 * * *$ & 0.712 & 0.031 & 12.033 \\
\hline & risk & - & - & - & - & - & - \\
\hline \multirow{2}{*}{$\begin{array}{l}\text { Two or more } \\
\text { adults without } \\
\text { dependent children }\end{array}$} & inc & $38.08^{* * *}$ & $265.86 * * *$ & $46.422 * * *$ & -0.163 & -0.272 & 9.691 \\
\hline & risk & $46.52 * * *$ & $480.59 * * *$ & $41.698 * * *$ & 0.949 & $0.450 * *$ & 9.269 \\
\hline \multirow{2}{*}{$\begin{array}{l}\text { Two or more } \\
\text { adults with } \\
\text { dependent children }\end{array}$} & inc & $49.87 * * *$ & $31.63 * * *$ & $39.720 * * *$ & -0.909 & -0.012 & 6.229 \\
\hline & risk & $68.98 * * *$ & $37.07 * * *$ & $19.377 * * *$ & 0.582 & -0.174 & 9.255 \\
\hline \multirow{2}{*}{$\begin{array}{l}\text { Three or more } \\
\text { adults }\end{array}$} & inc & $35.33 * * *$ & $42.72 * * *$ & $30.423 * * *$ & 0.582 & -0.252 & 12.498 \\
\hline & risk & $41.27 * * *$ & $277.63 * * *$ & $45.482 * * *$ & -0.167 & $0.395 * *$ & 6.564 \\
\hline \multirow{2}{*}{$\begin{array}{l}\text { Three or more } \\
\text { adults with } \\
\text { dependent children }\end{array}$} & inc & - & - & - & - & - & - \\
\hline & risk & $57.45 * * *$ & $899.47 * * *$ & $27.275 * * *$ & 0.371 & 0.053 & 9.022 \\
\hline \multirow{2}{*}{$\begin{array}{l}\text { Households } \\
\text { without dependent } \\
\text { children }\end{array}$} & $i n c$ & $47.07 * * *$ & $190.63 * * *$ & $77.970 * * *$ & 0.006 & 0.012 & 6.593 \\
\hline & risk & $34.41 * * *$ & $163.21 * * *$ & $39.214 * * *$ & 0.232 & -0.113 & 10.942 \\
\hline \multirow{2}{*}{$\begin{array}{l}\text { Households with } \\
\text { dependent children }\end{array}$} & inc & $45.89 * * *$ & $35.91 * * *$ & $77.043 * * *$ & -0.503 & -0.13 & 4.978 \\
\hline & risk & $68.73 * * *$ & $220.75 * * *$ & $35.899 * * *$ & 0.181 & -0.004 & 7.291 \\
\hline
\end{tabular}

The specification tests indicated the presence of: (a) heteroskedasticity; (b) panel first order autocorrelation in all models, except the WIND_GEG model; and (c) fixed effects. Accordingly, the specification tests suggested that the Driscoll and Kraay [44] estimator with fixed effects was suitable to handle those data features. The Driscoll and Kraay [44] is a covariance estimator, and it deals with small-samples considerably better than alternative traditional estimators, when heteroskedasticity and panel first order autocorrelation are present [45].

\section{Results and Discussion}

The results of the ARDL models are shown in Tables 6, 7, and 8. The Driscoll and Kraay estimator was used, and the parsimonious principle was followed. The Kao residual cointegration test and the JSE test revealed the existence of long-run relationships among variables in the models. The negative and highly statistically significant ECM values emphasise the confidence of the econometric results and reveal that the models are stable and able to return to the equilibrium after a disturbance. Besides, it should be highlighted that the ARDL method allows us to understand two 
important aspects. Firstly, the short-run reaction of the dependent variable is to variations or shocks in explanatory variables. This means, how the households' income and risk of poverty reacts in the short-run dynamics of adjustment to changes in the RES, natural gas consumption, and electricity prices. Secondly, the long-run equilibrium discloses how the households income and risk of poverty will converge if nothing is changed, namely in the explanatory variables $[41,46,47]$. The results show that on the equilibrium the majority of the effects is not desirable, mainly because of the increment of people at risk of poverty. Therefore, The energy transition towards RES and electricity should be well thought out, and the policy makers should change some policies and measures to not threaten the low-income households with energy poverty. 


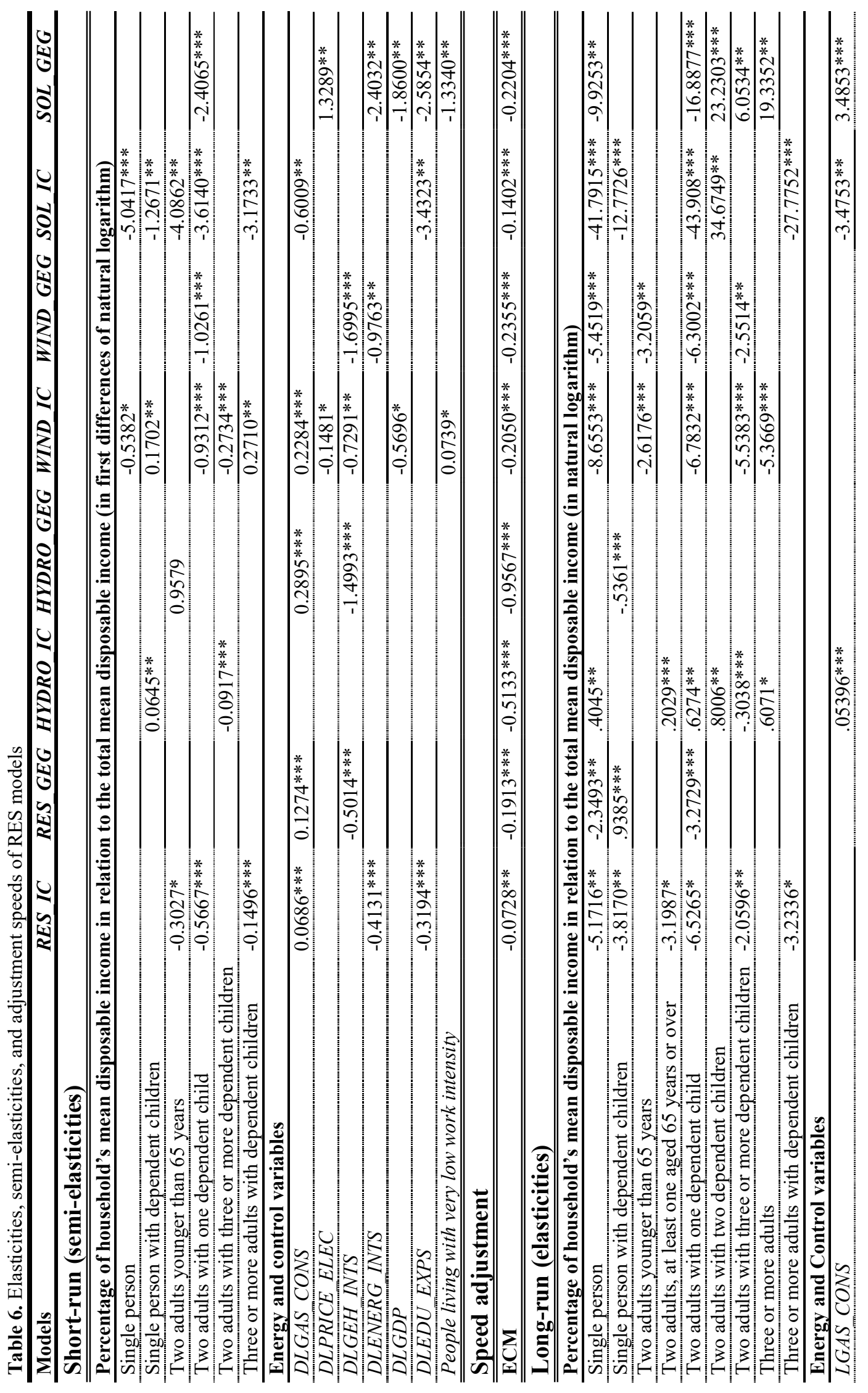




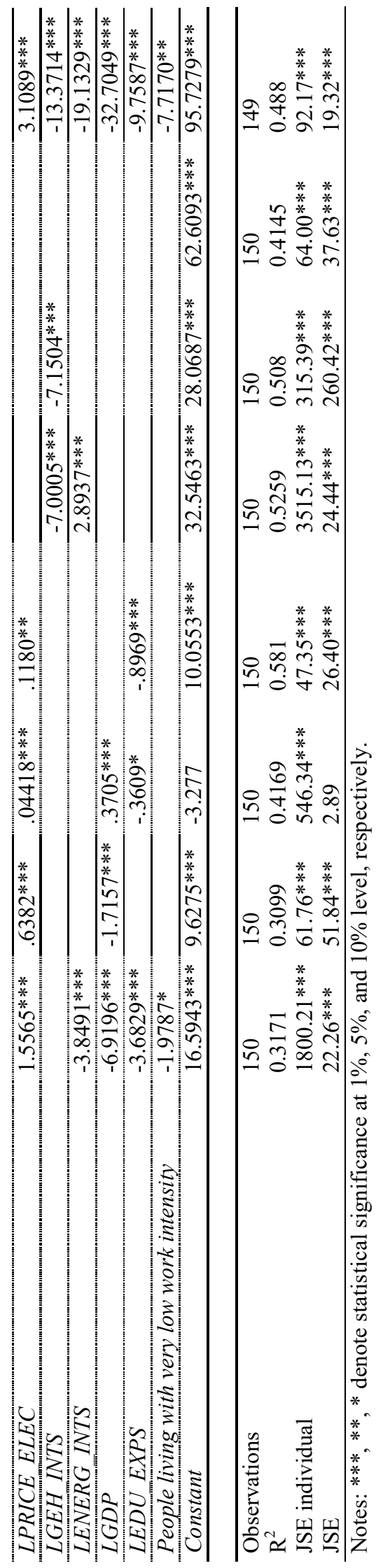


The results of the RES models highlight that: (a) income inequality may be delaying the deployment of RES, except for hydro power; (b) the income of large families has been effective in promoting solar PV; (c) energy intensity in economies has been the main driver of wind power deployment; and (d) no RES, other than wind power, need more subsidies. In fact, an increase of $1 \%$ in the price of residential electricity generates increases of $1.56 \%, 0.04 \%$, and $3.11 \%$, in the installed capacity of RES, hydro power, and solar PV generation, respectively. Both hydro power and solar PV could enter the market without being subsidised, due to household income, and electricity prices also have the potential to increase their deployment. Furthermore, the growing importance of hydro power and solar PV in electricity systems could further increase their competitiveness in the electricity market.

In households with children, there is empirical evidence for the following relationship; natural gas consumption decreases income and increases the risk of poverty, while higher electricity prices increase income and reduce the risk of poverty and social exclusion. In households without children, the contrary is shown to be true, contrary to what expected, that natural gas consumption decreases the risk of poverty, and higher electricity prices increase it. Electricity is accessible in the majority of cities, and metropolitan areas, and electricity can be used to power all home appliances. In contrast, access to natural gas is not always provided, and it can only be used for heating and cooking. Thus, the natural gas access excludes the consumption and use of households' appliances and electronic devices, which it is of growing importance on the society. Besides, home appliances for heating and cooking powered by electricity are safer than the home appliances powered by natural gas, which is an important concern for families with children. In fact, although studies are scarce, the literature shows that households with children generally consume more electricity and less natural gas than households without children [48-51]. The positive effect of children on electricity consumption could be due to greater use by children of certain appliances, such as televisions, personal computers, game consoles, etc. [48-51].

The frequent use of the internet by the children to access social media, entertainment, and learning processes, influenced by external social effects, and educational challenges could increase their propensity to have successful careers. Many parents also learn from their children to use the internet more effectively. The expansion of internet resources to work processes, and the increased capacity for processing its information could increase the income of households, further decreasing the risk of poverty. Furthermore, the access to more information and social media reduce the risk of info exclusion and, consequently, decrease the risk of poverty and social exclusion. Therefore, this effect of the internet and electricity on economies could increase the income of households, and enable better living condition, as suggested in our results. Policymakers should realize the importance of electricity consumption to households, and help those households threatened by poverty, or with a large number of dependent children. This aid could be provided by nonlinear electricity prices according to the number of family members and their risk of poverty, for example, by giving discount vouchers for electricity based on the number of dependent children, poverty and social status.

In households with children, electricity prices have been decreasing the risk of poverty, so, electricity is providing them with greater wellbeing. Furthermore, if these households joined DSM programs and energy efficiency measures, they could channel the resulting savings to other categories in their budgets. Thus, electricity could help them to avoid falling into the poverty trap and ensure greater wellbeing. Conversely, the transition of home appliances from natural gas to electricity must be well thought out 
for households without children. The price of electricity, which has been supporting the penetration of RES, is generally higher than the price of natural gas. During a transition to electricity, this type of household might divert funds from other categories, such as food and hygiene, to compensate for rising expenditure on energy. As such, this will decrease their productivity and future income, increasing the risk of their experiencing a poverty trap, or energy poverty. Therefore, their transition to electric home appliances should be planned to include DSM and energy efficiency programs to mitigate this negative effect, helping them reduce their electricity bills, and avoid falling into the poverty trap.

Wind power deployment and electricity generated from RES have a distinct effect on a household's risk of poverty. Wind power does not offer them high FiTs. Wind power is the largest contributor to the RES, and the deployment of wind power and its share of RES in the electricity mix has been diminishing the cost of electricity generation. During some periods, the high quantities of electricity generated from RES, mainly by wind power, have decreased the cost of generation below the RES surcharge. In fact, this price reduction could benefit households, if they have a time-of-use electricity tariff instead of a flat rate. Thus, it is crucial to incentivize consumers to use real-time electricity tariffs and DSM programs, and then adapt their consumption to periods when there is a high availability of natural resources. The households would then benefit from lower electricity prices, which would decrease their risk of poverty and social exclusion. 


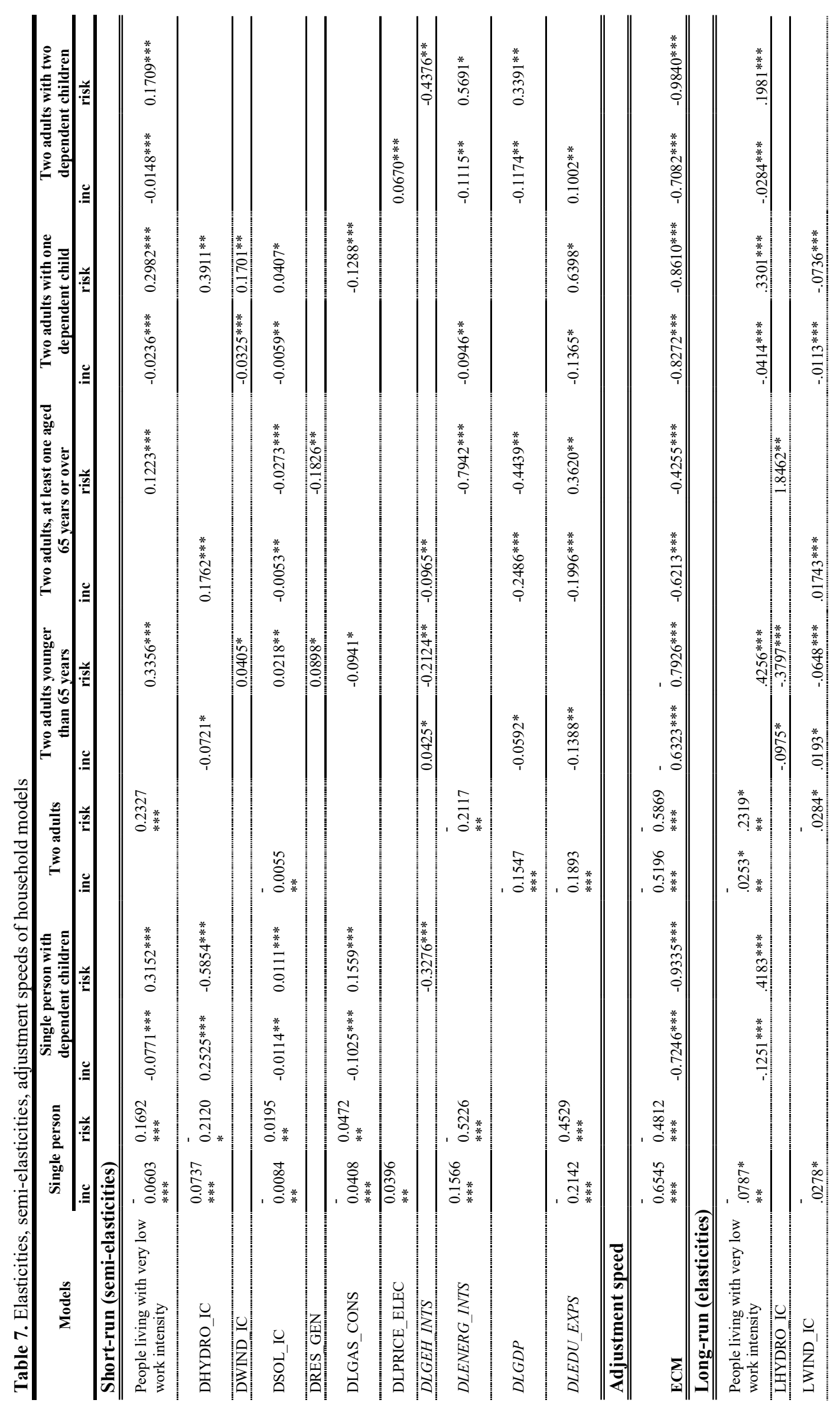




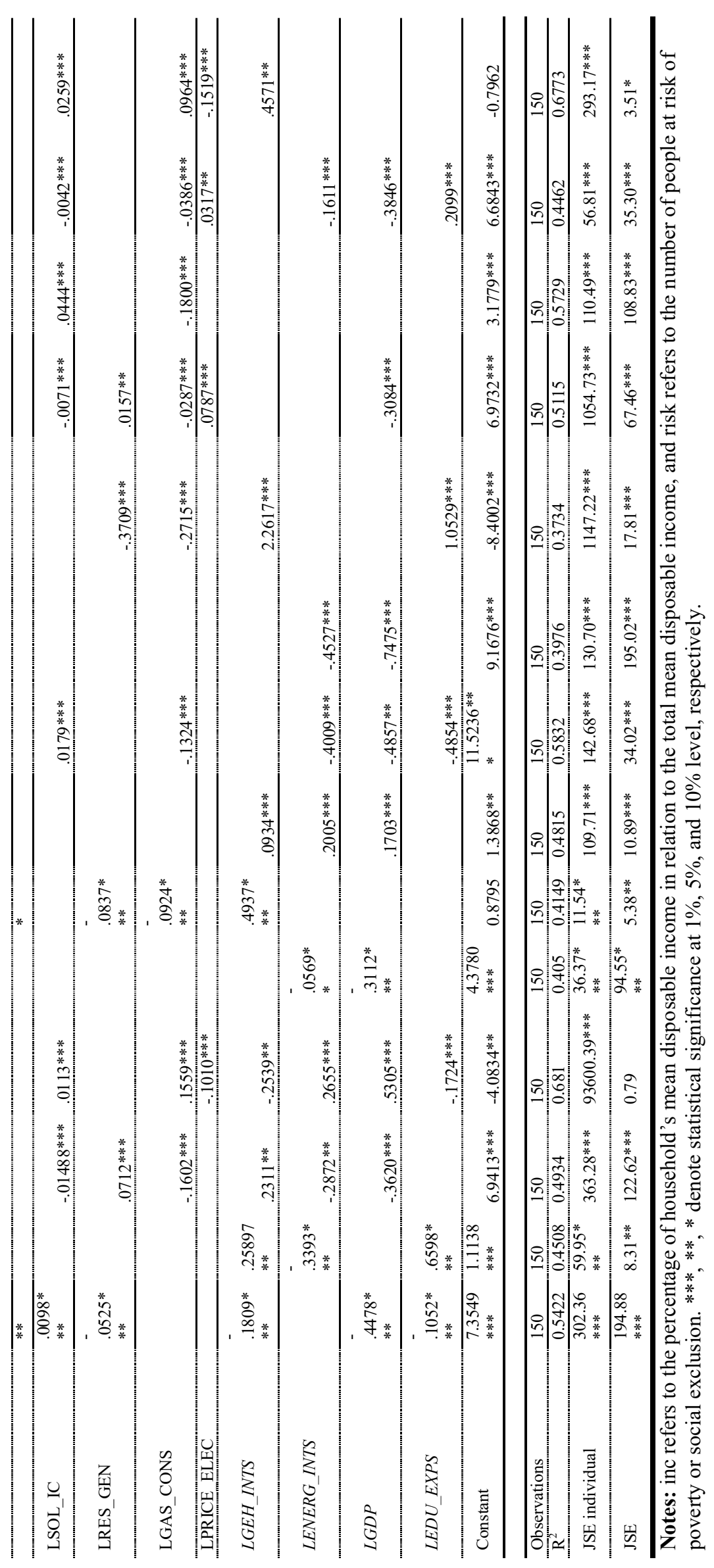




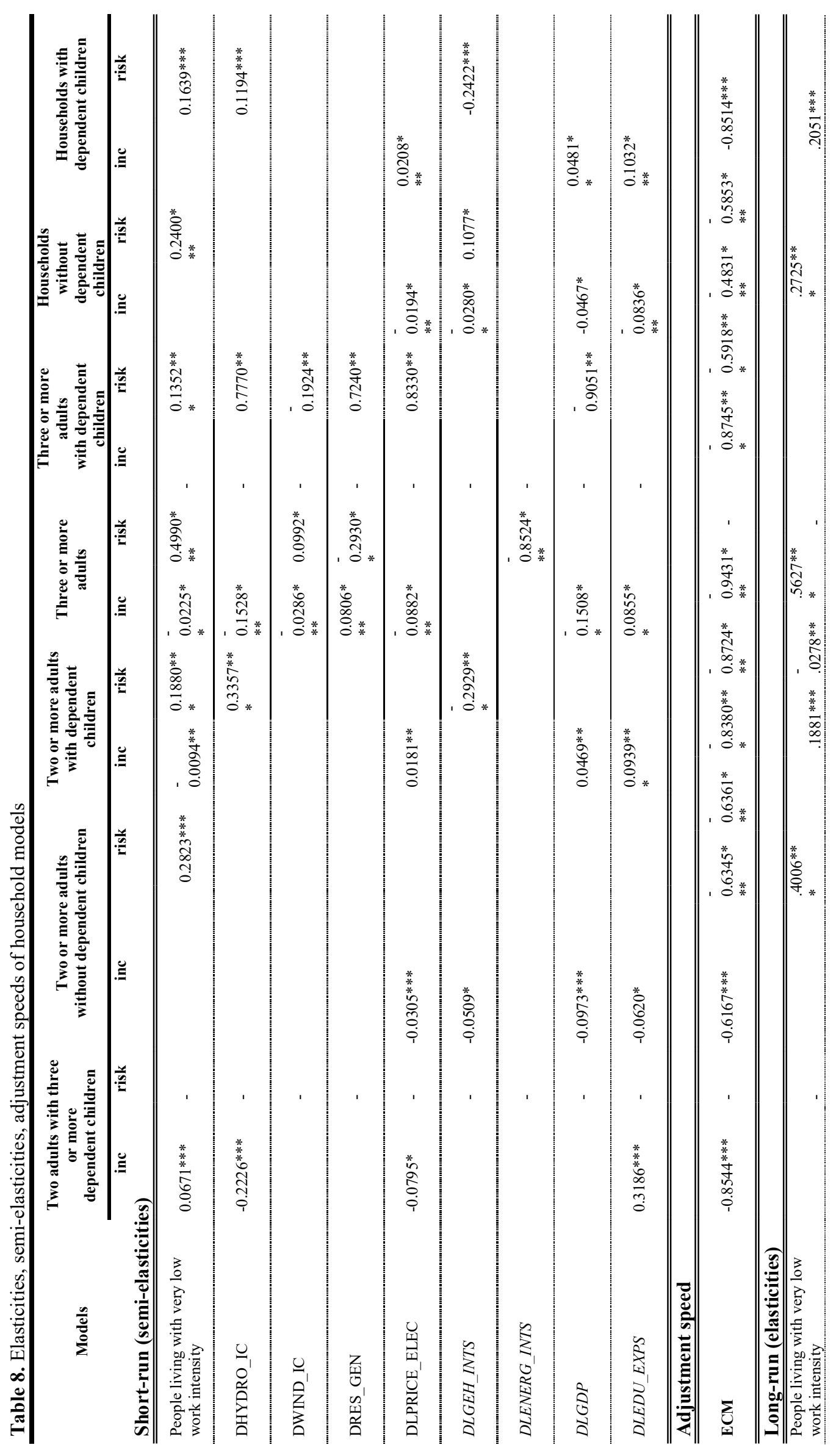




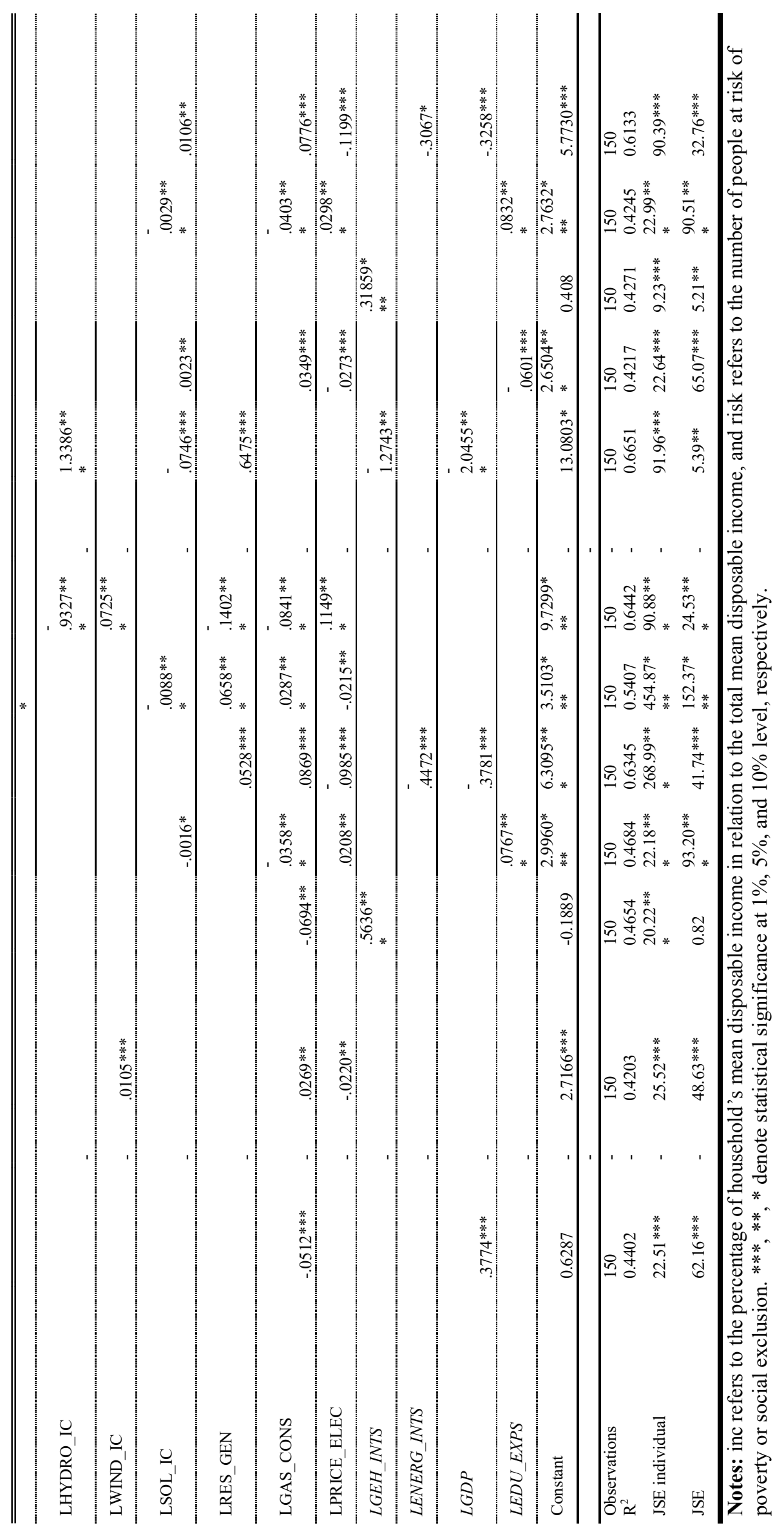


Solar PV deployment by major producers and auto-producers, has not yet produced the desired and expected effects, and has failed to positively affect economies and society overall. In fact, the installed capacity of solar PV has been decreasing incomes, and increasing the risk to households of poverty and social exclusion. This is an unexpected finding, and it deserves careful consideration. Firstly, this negative consequence could be a direct effect of the high FiTs awarded to promote solar PV. Indeed, the reduction of FiTs has not kept pace with reductions in the price of PV modules, which has led companies to install greater quantities of solar PV [7,18]. Consequently, the incentives to promote solar PV have permitted companies to benefit from higher returns, and, the economic benefits of RES deployment have not been shared with consumers, since the producers have retained all the profit.

Secondly, the majority of solar PV modules installed have been imported from non-European countries, such as China, Japan, and India. In fact, European solar panel producers have struggled to compete with non-European PV producers. For example, the price of PV panels produced in China was $€ 0.47 / \mathrm{Wp}$ (watt-peak) compared to the European average of $€ 1.10 / \mathrm{Wp}$ [52]. This led the European Commission to open antidumping investigations, and impose high trade restrictions to protect European PV manufactures [52]. However, several non-European manufactures and European PV companies have since merged. These international operations have been able to avoid the tariff restrictions, and enabled non-European modules to enter Europe at reduced prices [53]. Therefore, with manufacturing operations still being run outside of Europe, potential benefits to the European economy, such as job opportunities, have been cancelled out.

Currently, electricity prices and bills include the costs of generation, transportation, and RES surcharges. Thus, consumers have been paying for RES implementation and generation, along with the cost of providing fossil-fuel standby capacity to backup both RES and consumption peaks. In fact, this will keep increasing electricity prices, and consequently, the risk to households of poverty, including energy poverty. Households, particularly those on low-incomes, must be helped to support the cost of energy transition, and the economic surplus arising from energy transition has to be shared with consumers.

Incorporating RES is no longer a simple question of dispatch priority and guaranteed returns. In fact, households could play an important role in accommodating intermittent RES generation. In return, they should benefit from lower electricity prices. Thus, the economic surplus and wellbeing from using RES could be shared between consumers and producers. To enable households to enjoy a green electricity mix at reduced costs, governments and policy makers must be prepared to: (i) promote electricity conservation; (ii) subsidise more efficient home appliances instead of RES deployment; (iii) reward changes in consumption routines, through schemes such as electricity tariff differentiation; and (iv) encourage people to generate their own electricity, not through subsidies, but through low-interest rates. Tariff differentiation, if properly explained to consumers, could help them to adapt their consumption to periods with a higher availability of natural resources. Thus, it would reduce electricity prices and bills, and prevent sections of European society from falling into a poverty trap.

\section{Robustness check}


As additional proof of the results' robustness, a new framework, based on a long-run model, was performed. To perform a long-run model equation, the data must comply with two assumptions: (i) the variables included in the model are integrated of order one, i.e. I(1); and (ii) the series are cointegrated. The integration order test (Table 3 ) confirmed that all variables are I(1), and the Kao residual cointegration test (Table 4) corroborate that the variables are cointegrated. Accordingly, the data of this research complies with the two requirements needed to perform the long-run models. In the data used the endogeneity, residual cointegration, and serial correlation is suspicious, and the small sample bias is a concern. To surpass these econometric problems, the long-run models have been estimated using the Fully Modified Ordinary Least Squares (FMOLS), and Driscoll and Kraay estimators. The FMOLS estimator is suitable to handle sample size bias, serial correlation, and endogeneity [40]. Although the Driscoll and Kraay estimator, as mentioned before, is appropriate for handling sample size bias and serial correlation, instead of controlling endogeneity it controls heterogeneity between countries. In the FMOLS estimator the country fixed effects have been controlled manually, adding a dummy to each country. In the Driscoll and Kraay estimator the fixed effects are controlled automatically, choosing the option fixed effects. Therefore, the validity of ARDL long-run coefficients can be confirmed by the comparison with the coefficients of long-run models.

The long-run models results ${ }^{3}$ estimated by FMOLS and Driscoll and Kraay, validate the ARDL long-run coefficients, in terms of sign effects. This means that no change of signal effects between the two robustness estimators used and the ARDL models has been found. However, in terms of statistical significance, the long-run models estimated by Driscoll and Kraay reveal less statistically significant series, and different degrees of statistical significance when compared to either the ARDL models or the FMOLS results. The one main difference between the Driscoll and Kraay estimator and both the FMOLS estimator and the ARDL models, is the control of endogeneity. Thus, it is shown that, if endogeneity is not properly addressed, the results can be biased. The comparison of long-run ARDL and FMOLS results verified that the ARDL modelling deals effectively with the endogeneity issue, because of the similar results obtained. Therefore, the FMOLS estimator corroborated that the ARDL methodology was properly applied to the data used, and does not lead to biased results. In addition, the ARDL models permitted a breakdown of the total effects into short-run dynamics and long-run equilibrium, which enabled the retrieval of more conclusions.

\section{Conclusion}

This research focuses on the relationships between the deployment of RES and both the income of households and their risk of poverty. To evaluate the impact of RES implementation on society, the installed capacities have been divided into wind power, solar PV, and hydro power, and studied along with the share of electricity produced from RES, and electricity prices for the residential sector. In addition, households have also been classified according to EU-SILC categories, and empirically assessed by category. A Kao residual cointegration test, and an ARDL approach was employed to study fifteen EU countries, over a time-span from 2005 to 2015. The results show that RES deployment is directly linked with the living conditions of households. A battery

\footnotetext{
${ }^{3}$ They are not presented, but are available upon request to the authors.
} 
of model-specification tests was run, to choose the most suitable estimator, and to validate the long-run relationships. The long-run coefficients of ARDL models were subjected to a robustness check, corroborating the fact that the ARDL models have consistent and efficient parameter estimations. Furthermore, the robustness section highlights that endogeneity is present in the data used, and that it is properly addressed by the ARDL estimation.

In response to the questions proposed by this research it was found, firstly, that the income of different households has differing effects on RES promotion, benefiting hydro power, and solar PV. Secondly, the installed capacities of both wind power and hydro power, and the overall share of RES have dissimilar impacts on different households, but they have increased the income of some. However, the unexpected finding was the negative effect of solar PV deployment on household income. Thirdly, the capacity of wind and hydro power, and of all RES generation, have reduced the risk of poverty for some households, but have increased the risk for others. According to the income models for the period under analysis, solar PV has not yet been shown to reduce the risk of poverty. However, solar PV deployment by major players may have the potential to substitute fossil fuels during the day time, allowing for lower electricity prices. However, small-scale solar PV deployment, by consumers, could enhance the autonomy of their electricity consumption, and also improve these households' living conditions.

Access to modern and affordable electricity, through RES generation, will require an effort by society overall, particularly by consumers. Policymakers and electricity providers should raise people's awareness of the advantages of real-time tariffs over flat rates, and of self-generation through solar PV. They could encourage consumers to shift their consumption from peak periods, when electricity prices are higher, towards off-peak periods, when they will benefit from reduced prices. Furthermore, they could stimulate consumers to shift their consumption to periods with a higher availability of natural resources, by rewarding them with lower prices, and thus sharing the surplus of energy transition with them.

RES subsidies should be discontinued, and the savings used in cost-effective climate protection policies, or to subsidize household energy efficiency and saving measures. Indeed, the implementation and accommodation of RES should be accompanied by DSM programs, to involve consumers in the operation of RES, and share with them part of the economic surplus derived from the deployment of RES. Further research is needed to more precisely understand the impact of energy transition on household incomes and budgets, and how to mitigate any adverse consequences. Therefore, future research should focus on the impact of this transition on society, and also on policies, measures and programs, such as DSM and energy efficiency and saving, to overcome the negative implications of RES deployment. 


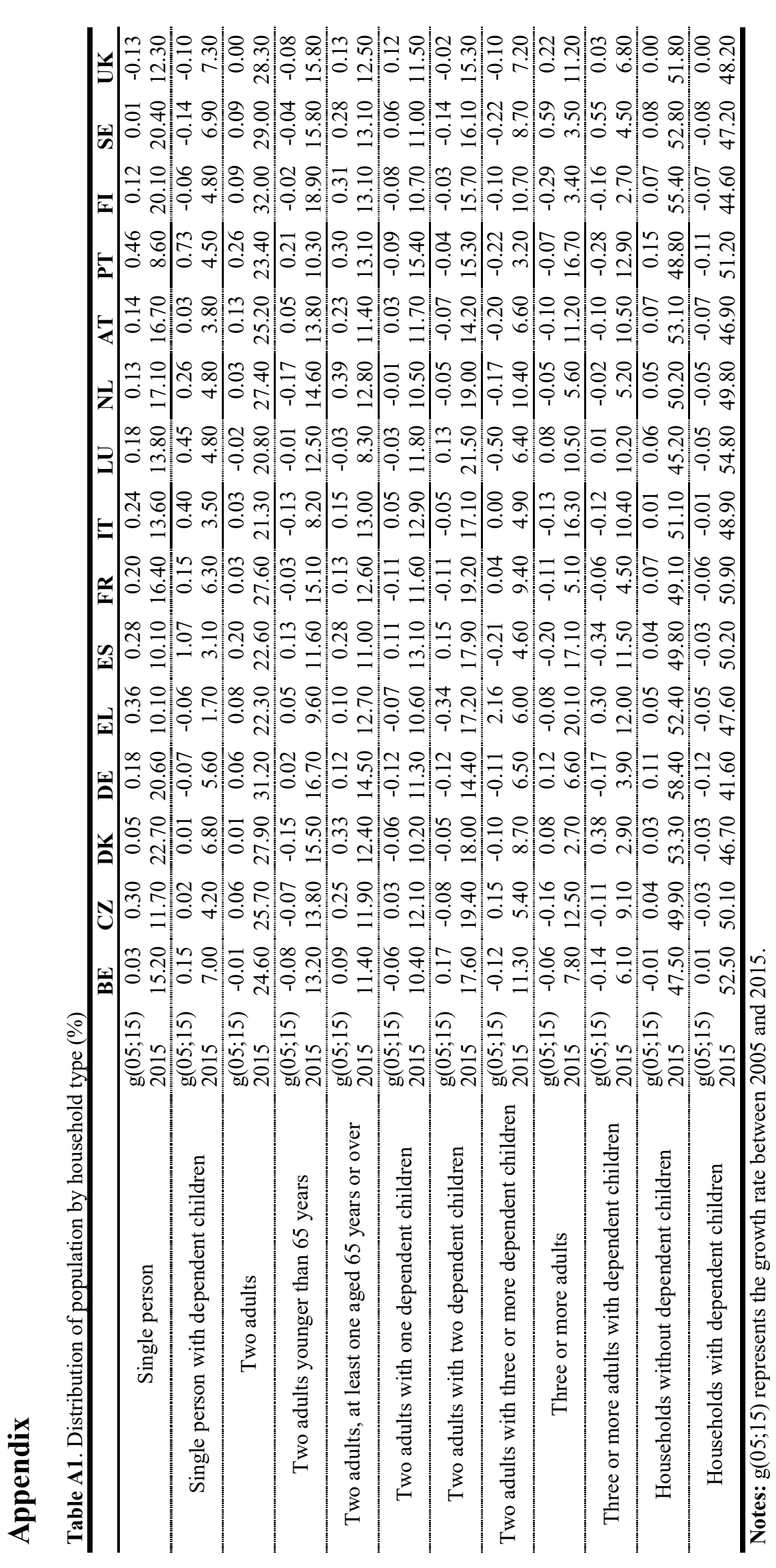




\section{References}

[1] United Nations. United Nations Millennium Development Goals. United Nations 2015.

[2] United Nations General Assembly. 70/1. Transforming our world: the 2030 Agenda for Sustainable Development. 2015.

[3] International Energy Agency. World Energy Outlook 2016 (Executive Summary). Iea Weo 2016. doi:http://www.iea.org/publications/freepublications/publication/WEB_WorldEn ergyOutlook2015ExecutiveSummaryEnglishFinal.pdf.

[4] UNDP. Energizing the Millennium Development Goals A Guide to Energy 's Role in Reducing Poverty. Development 2005:24.

[5] Shahbaz M, Sbia R, Hamdi H, Ozturk I. Economic growth, electricity consumption, urbanization and environmental degradation relationship in United Arab Emirates. Ecol Indic 2014;45:622-31. doi:10.1016/j.ecolind.2014.05.022.

[6] Omri A. An international literature survey on energy-economic growth nexus: Evidence from country-specific studies. Renew Sustain Energy Rev 2014;38:951-9. doi:10.1016/j.rser.2014.07.084.

[7] REN21. Renewables 2016-Global Status Report. 2016.

[8] Marques AC, Fuinhas JA. Are public policies towards renewables successful? Evidence from European countries. Renew Energy 2012;44:109-18. doi:10.1016/j.renene.2012.01.007.

[9] Aguirre M, Ibikunle G. Determinants of renewable energy growth: A global sample analysis. Energy Policy 2014;69:374-84. doi:10.1016/j.enpol.2014.02.036.

[10] Polzin F, Migendt M, Täube FA, von Flotow P. Public policy influence on renewable energy investments-A panel data study across OECD countries. Energy Policy 2015;80:98-111. doi:10.1016/j.enpol.2015.01.026.

[11] Hirth L, Ueckerdt F. Redistribution effects of energy and climate policy: The electricity market. Energy Policy 2013;62:934-47. doi:10.1016/j.enpol.2013.07.055.

[12] Nel WP, Cooper CJ. Implications of fossil fuel constraints on economic growth and global warming. Energy Policy 2009;37:166-80. doi:10.1016/j.enpol.2008.08.013.

[13] Glasnovic Z, Margeta J. Vision of total renewable electricity scenario. Renew Sustain Energy Rev 2011;15:1873-84. doi:10.1016/j.rser.2010.12.016.

[14] Flora R, Marques AC, Fuinhas JA. Wind power idle capacity in a panel of European countries. Energy 2014;66:823-30. doi:10.1016/j.energy.2013.12.061.

[15] Nelson T, Simshauser P, Kelley S. Australian Residential Solar Feed-in-Tariffs: Industry Stimulus or Regressive Form of Taxation. Econ Anal Policy 2011;41:113-29. doi:10.1016/S0313-5926(11)50015-3.

[16] Nelson T, Simshauser P, Nelson J. Queensland Solar Feed-In-Tariffs and the Merit-Order Effect: Economic benefit, or Regressive Taxation and Wealth Transfers. Econ Anal Policy 2012;42:277-301. doi:10.1016/S03135926(12)50030-5.

[17] Frondel M, Ritter N, Schmidt CM, Vance C. Economic impacts from the promotion of renewable energy technologies: The German experience. Energy Policy 2010;38:4048-56.

[18] Frondel M, Sommer S, Vance C. The burden of Germany's energy transition: An 
empirical analysis of distributional effects. Econ Anal Policy 2015;45:89-99. doi:10.1016/j.eap.2015.01.004.

[19] Andor M, Frondel M, Vance C. Installing Photovoltaics in Germany: A license to print money? Econ Anal Policy 2015;48:106-16. doi:10.1016/j.eap.2015.09.003.

[20] Valdés Lucas JN, Escribano Francís G, San Mart??n Gonzélez E. Energy security and renewable energy deployment in the EU: Liaisons Dangereuses or Virtuous Circle? Renew Sustain Energy Rev 2016;62:1032-46. doi:10.1016/j.rser.2016.04.069.

[21] Frondel M, Schmidt CM, Vance C. Revisiting Germany's solar cell promotion: An unfolding disaster. Econ Anal Policy 2014;44:3-13. doi:10.1016/j.eap.2014.02.001.

[22] Grösche P, Schröder C. On the redistributive effects of Germany's feed-in tariff. Empir Econ 2014;46:1339-83. doi:10.1007/s00181-013-0728-z.

[23] Jenner S, Groba F, Indvik J. Assessing the strength and effectiveness of renewable electricity feed-in tariffs in European Union countries. Energy Policy 2013;52:385-401. doi:10.1016/j.enpol.2012.09.046.

[24] Ketterer JC. The impact of wind power generation on the electricity price in Germany. Energy Econ 2014;44:270-80. doi:10.1016/j.eneco.2014.04.003.

[25] Kyritsis E, Andersson J, Serletis A. Electricity prices, large-scale renewable integration, and policy implications. Energy Policy 2017;101:550-60. doi:10.1016/j.enpol.2016.11.014.

[26] Frondel M, Ritter N, Schmidt CM. Germany's solar cell promotion: Dark clouds on the horizon. Energy Policy 2008;36:4198-204. doi:10.1016/j.enpol.2008.07.026.

[27] Pesaran MH. General Diagnostic Tests for Cross Section Dependence in Panels General Diagnostic Tests for Cross Section Dependence in Panels. SSRN Electron J 2004;1229.

[28] Hoyos RE De, Hoyos RE De, Sarafidis V, Sarafidis V. On Testing for Cross Sectional Dependence in Panel Data Models. Stata J 2006;6.

[29] Eberhardt M. Panel time-series modeling: New tools for analyzing xt data. 2011.

[30] Omri A, Nguyen DK. On the determinants of renewable energy consumption: International evidence. Energy 2014;72:554-60. doi:10.1016/j.energy.2014.05.081.

[31] Romano AA, Scandurra G, Carfora A, Fodor M. Renewable investments: The impact of green policies in developing and developed countries. Renew Sustain Energy Rev 2017;68:738-47. doi:10.1016/j.rser.2016.10.024.

[32] Neumayer E, Plumper T. Inequalities of income and inequalities of longevity: A cross-country study. Am J Public Health 2016;106:160-5. doi:10.2105/AJPH.2015.302849.

[33] Page L, Goldstein DG. Subjective beliefs about the income distribution and preferences for redistribution. Soc Choice Welfare 2016;47:25-61. doi:10.1007/s00355-015-0945-9.

[34] Gęstwicki FE, Wędrowska E. Assessment of the Degree of the Divergence and Inequality of Household Income Distribution in Poland in the Years 2005-2013. Folia Oeconomica Stetin 2016;16. doi:10.1515/foli-2016-0004.

[35] Turnovsky SJ. Economic growth and inequality: The role of public investment. J Econ Dyn Control 2015;61:204-21. doi:10.1016/j.jedc.2015.09.009.

[36] Gutierrez L. On the power of panel cointegration tests: A Monte Carlo comparison. Econ Lett 2003. doi:10.1016/S0165-1765(03)00066-1.

[37] Fuinhas JA, Marques AC, Couto AP. Oil-Growth Nexus in Oil Producing 
Countries: Macro Panel Evidence. Int J Energy Econ Policy 2015;5:148-63.

[38] Papageorgiou T, Michaelides PG, Tsionas EG. Business cycle determinants and fiscal policy: A Panel ARDL approach for EMU. J Econ Asymmetries 2016;13:57-68. doi:10.1016/j.jeca.2015.12.001.

[39] Pesaran MH, Shin Y. Pesaran, M. H., \& Shin, Y. (1998). An autoregressive distributed-lag modelling approach to cointegration analysis. Econom Soc Monogr 1999;31:371-413. doi:10.1017/CCOL521633230.

[40] Phillips P, Hansen B. Statistical Inference in Instrumental Variables Regression with I(1) Processes. Rev Econ Stud 1990;57:99-125.

[41] Marques AC, Fuinhas JA, Neves SA. Ordinary and Special Regimes of electricity generation in Spain: How they interact with economic activity. Renew Sustain Energy Rev 2018;81:1226-40. doi:10.1016/j.rser.2017.09.006.

[42] Marques AC, Fuinhas JA, Pereira DA, Fontes JW. Is renewable energy penetration preserving the fossil fuels dependency? An empirical assessment, 2017.

[43] Rafindadi AA, Ozturk I. Impacts of renewable energy consumption on the German economic growth: Evidence from combined cointegration test. Renew Sustain Energy Rev 2017;75:1130-41. doi:10.1016/j.rser.2016.11.093.

[44] Driscoll JC, Kraay AC. Consistent Covariance Matrix Estimation with Spatially Dependent Panel Data. Rev Econ Stat 1998;80:549-60.

[45] Hoechle D. Robust standard errors for panel regressions with cross-sectional dependence. Stata J 2007;7:281-312.

[46] Marques AC, Fuinhas JA, Pereira DA. Have fossil fuels been substituted by renewables? An empirical assessment for 10 European countries. Energy Policy 2018;116. doi:10.1016/j.enpol.2018.02.021.

[47] Pesaran MH, Shin Y. Econometrics and Economic Theory in the 20th Century. Econom Soc Monogr 1999;31:371-413. doi:10.1017/CCOL521633230.

[48] Brounen D, Kok N, Quigley JM. Residential energy use and conservation: Economics and demographics. Eur Econ Rev 2012;56:931-45. doi:10.1016/j.euroecorev.2012.02.007.

[49] Chalal ML, Benachir M, White M, Shahtahmassebi G, Cumberbatch M, Shrahily R. The impact of the UK household life-cycle transitions on the electricity and gas usage patterns. Renew Sustain Energy Rev 2017;80:505-18. doi:10.1016/j.rser.2017.05.222.

[50] Longhi S. Residential energy use and the relevance of changes in household circumstances. ISER Work Pap Ser 2014.

[51] Shirani F, Parkhillb K, Butler C, Groves C, Pidgeon N, Henwood K. Asking about the future: methodological insights from energy biographies. Int J Soc Res Methodol 2016;19:429-44. doi:10.1080/13645579.2015.1029208.

[52] ProSun. Dumping. EU ProSun 2016. http://www.prosun.org/en/tradedistortions/\#toggle-id-1.

[53] McCarthy KJ. On the influence of the European trade barrier on the Chinese pv industry: Is the solution to the solar-dispute "successful"? Energy Policy 2016;99:154-7. doi:10.1016/j.enpol.2016.09.055. 